\title{
Rapid and Automatic Zero-Offset Calibration of a 2-DOF Parallel Robot Based on a New Measuring Mechanism
}

\author{
Jiangping Mei - Jiawei Zang - Yabin Ding*- Shenglong Xie - Xu Zhang \\ Ministry of Education, Tianjin University, Key Laboratory of Mechanism Theory and Equipment Design, China
}

This paper deals with the rapid and automatic zero-offset calibration of a 2-DOF parallel robot using distance measurements. The calibration system is introduced with emphasis on the design of a new measuring mechanism. A simplified error model of the robot is proposed after the sensitivity analyses of source errors, based on which a zero-offset identification model is developed using the truncated singular value decomposition (TSVD) method, and then it is modified with the manufacturing and assembly errors of the measuring mechanism (MAEMM). Furthermore, an optimization approach for selecting measurement positions is proposed by considering the condition number of the identification matrix. Finally, simulations and experiments are carried out to verify the effectiveness of the zero-offset calibration method. The results show that the identification model has good identifiability and robustness, and the position accuracy after calibration can be significantly improved.

Keywords: parallel robot, calibration, zero offset, measuring mechanism

Highlights

- A new measuring mechanism is designed for the zero-offset calibration of a 2-DOF parallel robot.

- The zero-offset identification model is truncated by the TSVD method and modified with the MAEMM.

- The measurement positions are optimized based on the condition number of the identification matrix.

- Simulations and experiments are carried out to verify the effectiveness of the calibration method.

\section{O INTRODUCTION}

Parallel robots have been widely used in many fields. This can be exemplified by the well-known Delta robot [1], including many applications of its modified versions, [2] to [4]. In recent years, the 2-DOF translational parallel robots has drawn ongoing interest from academia and industry due to their compact configurations and high stiffness, such as the very successful 4-PP [5] and 4-PP-E [6] simple decoupled XY parallel robots with enhanced stiffness, the Diamond [7] for high speed operation and the large-workspace 2-DOF parallel robot for solar tracking systems [8].

Position errors of parallel robots are mainly caused by their zero offsets, i.e. the errors between the nominal and actual initial positions of active links (see Fig. 1), provided that adequate fundamental geometric accuracy can be achieved at the manufacturing and assembly levels, [9] and [10]. The zero offsets may be caused by the control faults, collisions, or looseness of active joints at any time in practical applications. Therefore, to ensure the position accuracy, it is necessary to eliminate the zero offsets when they occur.

It is well recognized that the zero-offset calibration, one of the kinematic calibrations, is a practical and economical way to reduce zero offsets, [11] and [12]. The zero-offset calibration pays more attention to the calibration of the zero offsets than the geometric errors. Furthermore, a fine calibration of the zero offsets is the premise to ensure the calibration accuracy of the geometric errors [13]. In general, the calibration can be implemented by four sequential processes, i.e. error modelling, measurement, identification and compensation such that the zero offsets affecting the position accuracy can be suppressed [14].
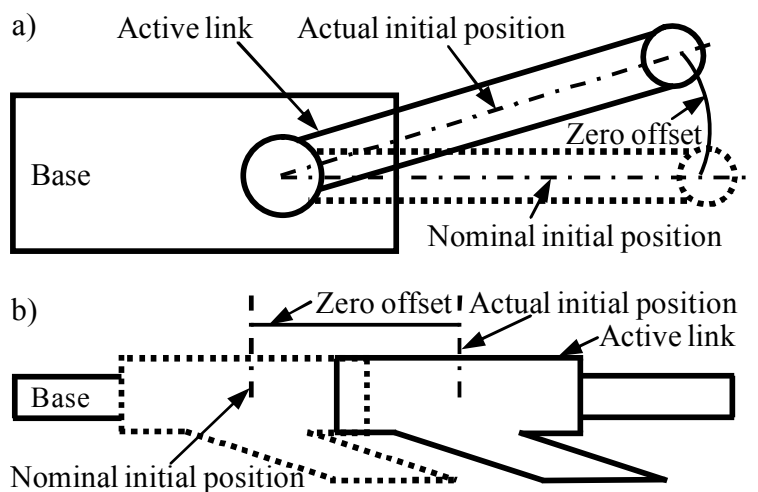

Fig. 1. Zero offsets of parallel robots; a) parallel robot with revolute joint; and b) parallel robot with prismatic joint

The methods of the zero-offset calibration can be classified into self/autonomous calibration [15] and external calibration [16]. Compared with the self/autonomous calibration that realizes the 
identification of the zero offsets through minimizing the discrepancies between the measured and computed values of joint space sensors, the external calibration finishes the same work using task space sensors. Furthermore, the external calibration can be classified into the coordinate-based approach and the distance-based approach, [17] to [20]. In comparison with the coordinate-based approach, the advantage of the distance-based approach lies in that it is invariant with the chosen reference frame. Hence, it has been widely applied for the calibrations. For the data acquisition during the measurement process, it is usually implemented using a large metrology device, e.g. a laser tracker or interferometer, which is costly and inconvenient to use. Meanwhile, to ensure the identifiability, the number of measurement positions usually tends to be overlarge, which reduces the measurement efficiency. Therefore, the problem of how to make the measurement process in a time and cost-effective manner needs to be further studied.

The identification is the kernel process of calibration, and it is usually implemented using the least square (LS) method [21]. However, if the zero offsets are identified together with too many geometric errors, it may lead to a sharp increase in the condition number of the identification matrix and thereby cause the nonlinear ill-conditioning problem for identification model. To solve this problem, the ridge estimation (RE) method and the truncated singular value decomposition (TSVD) method have been widely adopted [22] and [23]. Some studies have indicated that the TSVD has better identification accuracy than the LS does, and it is easier to implement than the RE is. Though the nonlinear illconditioning problem can be solved to some extent by the RE or TSVD, the problem of how to further improve the identification accuracy of the zero offsets needs to be thoroughly investigated.

This paper deals with the rapid and automatic zero offset calibration of a 2-DOF parallel robot [24]. We focus on: 1) the design of a new measuring mechanism to make the measurement process in a time and cost-effective manner; 2) the development of a simplified error model containing the zero offsets of the robot; 3) the development of an identification method to solve the nonlinear ill-conditioning problem and improve the identifiability; 4) the selection of optimal measurement positions to further improve the identifiability and the measurement efficiency. Simulations and experiments are also carried out to validate the proposed calibration method.

\section{SYSTEM DESCRIPTION}

As shown in Fig. 2, the 2-DOF parallel robot is revolute jointed. Driven by two active proximal links, the robot can provide its moving platform with a 2-DOF translational moving capability.

Fig. 3 shows the new measuring mechanism which mainly consists of two revolute joints, two guide rods, a shipper rod and a linear scale. The two guide rods and the linear scale are arranged in parallel and fixed on two connecting plates. The shipper rod and the reading head of the linear scale are fixed on a slider which is vertically connected to the two guide rods by linear bearings. The revolute joints 3 and 4 are fixed on the upper connecting plate and the end of the shipper rod, respectively, based on which the measuring mechanism can be connected to the base and the moving platform of the robot.

By letting the moving platform undergo several measurement positions, the distance changes between the revolute joints 3 and 4 can be automatically obtained by the reading head and then transferred into the zero-offset calibration model in the robot controller. Thus, the zero offsets can be rapidly calibrated.

1 - Revolute joint 1

2 - Revolute joint 2

3 - Passive proximal link

4 - Active proximal link

5 - Rotation shaft 1

6 - Rotation shaft 2

7 - Distal links

8 - Measuring mechanism

9 - Moving platform

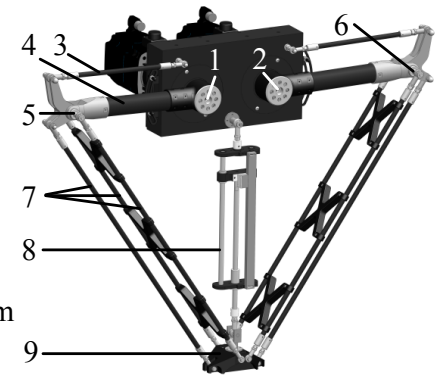

Fig. 2. 3D model of the 2-DOF parallel robot

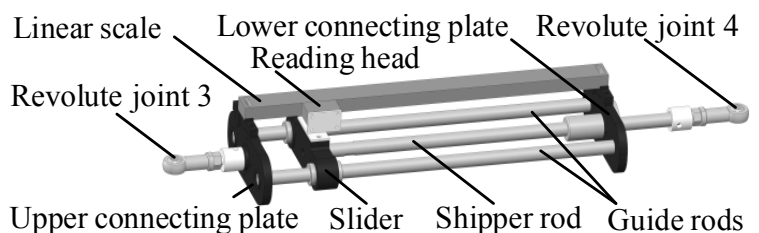

Fig. 3. 3D model of the measuring mechanism

\section{KINEMATIC ANALYSES}

The 2-DOF parallel robot can be simplified as shown in Fig. 4. In the $O-x y$ coordinate system, the nominal position vector, $\boldsymbol{r}=(x y)^{\mathrm{T}}$, of the reference point $P$ can be written as:

$$
\boldsymbol{r}=\boldsymbol{e}_{i}+L_{i} \boldsymbol{u}_{i}+l_{i} \boldsymbol{w}_{i}, i=1,2,
$$


where $L_{i}, l_{i}, \boldsymbol{u}_{i}$ and $\boldsymbol{w}_{i}$ are the nominal lengths and nominal unit orientation vectors of the proximal and distal links, respectively; $\boldsymbol{e}_{i}$ is the nominal position vector of $A_{i}$; and

$$
\begin{aligned}
& \boldsymbol{u}_{i}=\left(\begin{array}{lll}
\cos \theta_{i} & \sin \theta_{i}
\end{array}\right)^{\mathrm{T}}, \quad \boldsymbol{w}_{i}=\left(\begin{array}{ll}
\cos \varphi_{i} & \sin \varphi_{i}
\end{array}\right)^{\mathrm{T}}, \\
& \boldsymbol{e}_{i}=\left(\begin{array}{ll}
e_{i x} & e_{i y}
\end{array}\right)^{\mathrm{T}},
\end{aligned}
$$

where $\theta_{i}$ and $\varphi_{i}$ are the nominal rotation angles of the proximal and distal links, respectively.

Taking 2-norm on the two sides of Eq. (1), the solution of the inverse positional analysis can then be expressed as:

$$
\theta_{i}=2 \arctan \frac{-C_{i}-\sqrt{C_{i}^{2}-D_{i}^{2}+E_{i}^{2}}}{D_{i}-E_{i}},
$$

where $C_{i}=-2 L_{i}\left(y-e_{i y}\right), \quad D_{i}=-2 L_{i}\left(x-e_{i x}\right)$, $E_{i}=\left(x-e_{i x}\right)^{2}+\left(y-e_{i y}\right)^{2}+L_{i}^{2}-l_{i}^{2}$.

Hence, $\boldsymbol{w}_{i}$ and the position vector from $O_{1}$ to $O_{2}$, denoted by $\lambda$, can be calculated as follows:

$$
\boldsymbol{w}_{i}=\frac{\boldsymbol{r}-\boldsymbol{e}_{i}-L_{i} \boldsymbol{u}_{i}}{l_{i}}, \lambda=\boldsymbol{r}-\boldsymbol{c}-\boldsymbol{d},
$$

where $c$ is the position vector from $O$ to $O_{1} ; \boldsymbol{d}$ is the position vector from $O_{2}$ to $P$.

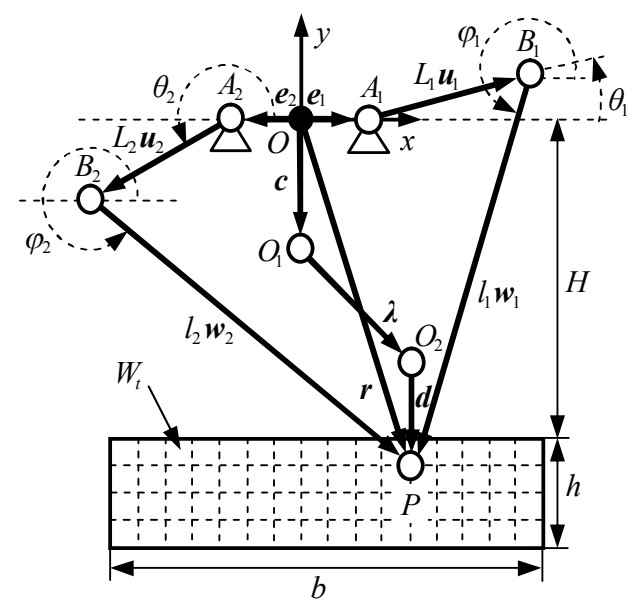

Fig. 4. Kinematic model of the 2-DOF parallel robot (Note: $A_{1}\left(A_{2}\right)$ is the nominal rotation centre of the revolute joint $1(2)$;

$B_{1}\left(B_{2}\right)$ is the nominal rotation centre of the rotation shaft $1(2)$;

$\mathrm{O}_{1}\left(\mathrm{O}_{2}\right)$ is the nominal rotation centre of the revolute joint $3(4)$;

$P$ is a reference point at the centre of the moving platform; $W_{t}$ is the workspace; $H$ is the distance between 0 and the upper boundary of the workspace; $h$ is the height of the workspace; $b$ is the width of the workspace)

To develop the forward positional model, rewrite Eq. (1) as:

$$
\boldsymbol{r}^{\mathrm{T}} \boldsymbol{r}-2\left(\boldsymbol{e}_{i}+L_{i} \boldsymbol{u}_{i}\right)^{\mathrm{T}} \boldsymbol{r}+\left(\boldsymbol{e}_{i}+L_{i} \boldsymbol{u}_{i}\right)^{\mathrm{T}}\left(\boldsymbol{e}_{i}+L_{i} \boldsymbol{u}_{i}\right)=l_{i}^{2} .
$$

Subtract the two equations in Eq. (5) with each other yields:

$$
x=-\frac{M y+S}{F},
$$

where

$$
\begin{aligned}
& F=2\left[\left(\boldsymbol{e}_{2}+L_{2} \boldsymbol{u}_{2}\right)^{\mathrm{T}}-\left(\boldsymbol{e}_{1}+L_{1} \boldsymbol{u}_{1}\right)^{\mathrm{T}}\right] \boldsymbol{a}_{1}, \\
& M=2\left[\left(\boldsymbol{e}_{2}+L_{2} \boldsymbol{u}_{2}\right)^{\mathrm{T}}-\left(\boldsymbol{e}_{1}+L_{1} \boldsymbol{u}_{1}\right)^{\mathrm{T}}\right] \boldsymbol{a}_{2}, \\
& S=\left\|\boldsymbol{e}_{1}+L_{1} \boldsymbol{u}_{1}\right\|^{2}-\left\|\boldsymbol{e}_{2}+L_{2} \boldsymbol{u}_{2}\right\|^{2}-\left(l_{1}^{2}-l_{2}^{2}\right), \\
& \boldsymbol{a}_{1}=\left(\begin{array}{ll}
1 & 0
\end{array}\right)^{\mathrm{T}}, \quad \boldsymbol{a}_{2}=\left(\begin{array}{ll}
0 & 1
\end{array}\right)^{\mathrm{T}} .
\end{aligned}
$$

Substitute Eq. (6) into Eq. (5), then the quadratic equation of $y$ can be written as:

$$
N y^{2}+Q_{i}+R_{i}=0,
$$

where

$$
\begin{aligned}
& R_{i}=\frac{S^{2}}{F^{2}}+2 \frac{S}{F}\left(\boldsymbol{e}_{i}+L_{i} \boldsymbol{u}_{i}\right)^{\mathrm{T}} \boldsymbol{a}_{1}+\left\|\boldsymbol{e}_{i}+L_{i} \boldsymbol{u}_{i}\right\|^{2}-l_{i}^{2}, \\
& N=1+\frac{M^{2}}{F^{2}}, \quad Q_{i}=\frac{2 M S}{F^{2}}+2\left(\boldsymbol{e}_{i}+L_{i} \boldsymbol{u}_{i}\right)^{\mathrm{T}}\left(\frac{M}{F} \boldsymbol{a}_{1}-\boldsymbol{a}_{2}\right) .
\end{aligned}
$$

According to the assembly mode of the robot, the $y$ coordinate of $P$ can be expressed as:

$$
y=\frac{-Q_{i}-\sqrt{Q_{i}^{2}-4 N R_{i}}}{2 N} .
$$

Hence, substitute Eq. (8) into Eq. (6), then the $x$ coordinate of $P$ can be determined.

\section{ERROR MODELLING AND SENSITIVITY ANALYSES}

The first-order approximation of Eq. (1) can be formulated by:

$$
\Delta \boldsymbol{r}=\Delta \boldsymbol{e}_{i}+\Delta L_{i} \boldsymbol{u}_{i}+L_{i} \Delta \boldsymbol{u}_{i}+\Delta l_{i} \boldsymbol{w}_{i}+l_{i} \Delta \boldsymbol{w}_{i},
$$

where $\Delta \boldsymbol{r}=(\Delta x \Delta y)^{\mathrm{T}}$ is the position error vector of the reference point $P ; \Delta \boldsymbol{e}_{i}=\left(\Delta e_{i x} \Delta e_{i y}\right)^{\mathrm{T}}$ is the position error vector of $A_{i} ; \Delta L_{i}, \Delta l_{i}, \Delta \boldsymbol{u}_{i}$ and $\Delta \boldsymbol{w}_{i}$ are the length errors and orientation error vectors of the proximal and distal links. Furthermore, the first-order approximation of $\boldsymbol{u}_{i}$ can be written as:

$$
\Delta \boldsymbol{u}_{i}=\Delta \theta_{i}\left(-\sin \theta_{i} \cos \theta_{i}\right)^{\mathrm{T}}=\boldsymbol{Q} \boldsymbol{u}_{i} \Delta \theta_{i}, \boldsymbol{Q}=\left[\begin{array}{cc}
0 & -1 \\
1 & 0
\end{array}\right],
$$

where $\Delta \theta_{i}$ is the zero offset of the robot.

Then, taking the dot product with $\Delta \boldsymbol{w}_{i}^{\mathrm{T}}$ on the both sides of Eq. (9) (note that $\boldsymbol{w}_{i} \perp \Delta \boldsymbol{w}_{i}$ ) yields:

$$
\boldsymbol{w}_{i}^{\mathrm{T}} \Delta \boldsymbol{r}=\boldsymbol{w}_{i}^{\mathrm{T}} \Delta \boldsymbol{e}_{i}+\Delta L_{i} \boldsymbol{w}_{i}^{\mathrm{T}} \boldsymbol{u}_{i}+L_{i} \boldsymbol{w}_{i}^{\mathrm{T}} \boldsymbol{Q} \boldsymbol{u}_{i} \Delta \theta_{i}+\Delta l_{i} .
$$

For a parallel robot, its error model is usually expressed in matrix form, such that the relationship between the position error and the source errors can be directly revealed by an error transfer matrix. 
According to Eq. (11), the error model of the robot can be expressed as

$$
\Delta \boldsymbol{r}=\boldsymbol{J}^{\prime} \Delta \boldsymbol{q}^{\prime},
$$

where $\boldsymbol{J}^{\prime}$ denotes the error transfer matrix, and

$$
\begin{aligned}
& \boldsymbol{J}^{\prime}=\left[\begin{array}{ll}
\boldsymbol{w}_{1} & \boldsymbol{w}_{2}
\end{array}\right]^{-\mathrm{T}}\left[\begin{array}{cc}
\boldsymbol{J}_{1}^{\prime} & \mathbf{0} \\
\mathbf{0} & \boldsymbol{J}_{2}^{\prime}
\end{array}\right], \quad \Delta \boldsymbol{q}^{\prime}=\left(\begin{array}{c}
\Delta \boldsymbol{q}_{1}^{\prime} \\
\Delta \boldsymbol{q}_{2}^{\prime}
\end{array}\right), \\
& \boldsymbol{J}_{1}^{\prime}=\left(\begin{array}{lllll}
\boldsymbol{w}_{1}^{\mathrm{T}} \boldsymbol{Q} \boldsymbol{\boldsymbol { u } _ { 1 }} & w_{1 x} & w_{1 y} & \boldsymbol{w}_{1}^{\mathrm{T}} \boldsymbol{u}_{1} & 1
\end{array}\right), \\
& \boldsymbol{J}_{2}^{\prime}=\left(\begin{array}{lllll}
\boldsymbol{w}_{2}^{\mathrm{T}} \boldsymbol{Q} \boldsymbol{u}_{2} & w_{2 x} & w_{2 y} & \boldsymbol{w}_{2}^{\mathrm{T}} \boldsymbol{u}_{2} & 1
\end{array}\right), \\
& \Delta \boldsymbol{q}_{1}^{\prime}=\left(\begin{array}{lllll}
L_{1} \Delta_{1} & \Delta e_{1 x} & \Delta e_{1 y} & \Delta L_{1} & \Delta l_{1}
\end{array}\right)^{\mathrm{T}}, \\
& \Delta \boldsymbol{q}_{2}^{\prime}=\left(\begin{array}{lllll}
L_{2} \Delta_{s_{2}} & \Delta e_{2 x} & \Delta e_{2 y} & \Delta L_{2} & \Delta l_{2}
\end{array}\right)^{\mathrm{T}} .
\end{aligned}
$$

Since the robot has symmetrical geometry, the sensitivity analyses of the source errors can be studied by analysing the variation of $\Delta \rho_{0}\left(\Delta \rho_{0}\right.$ is the absolute distance error of $P_{0}$, and $P_{0}$ is the home position at which $\theta_{1}=0^{\circ}$ and $\theta_{2}=180^{\circ}$ ) versus the source errors within the 1 st limb.

Given $L_{1}=L_{2}=L, l_{1}=l_{2}=l, e_{1 x}=-e_{2 x}=e_{x}$ and $e_{1 y}=e_{2 y}=e_{y}$, the nominal geometric parameters of the robot are listed in Table 1, and the results of the sensitivity analyses are presented in Fig. 5. It can be seen that the position accuracy is more sensitive to the zero offset than the geometric errors. Hence assume that the adequate fundamental geometric accuracy of the robot can be achieved, Eq. (12) can be simplified as follows:

$$
\Delta \boldsymbol{r}=\boldsymbol{J} \Delta \boldsymbol{q},
$$

where

$$
\boldsymbol{J}=\left[\begin{array}{ll}
\boldsymbol{w}_{1} & \boldsymbol{w}_{2}
\end{array}\right]^{-\mathrm{T}}\left[\begin{array}{cc}
\boldsymbol{w}_{1}^{\mathrm{T}} \boldsymbol{Q} \boldsymbol{u}_{1} & 0 \\
0 & \boldsymbol{w}_{2}^{\mathrm{T}} \boldsymbol{Q} \boldsymbol{u}_{2}
\end{array}\right], \Delta \boldsymbol{q}=\left(\begin{array}{c}
L_{1} \Delta \theta_{1} \\
L_{2} \Delta \theta_{2}
\end{array}\right) .
$$

\section{Table 1. Nominal geometric parameters [mm]}

\begin{tabular}{ccccccccccc}
\hline$e_{x}$ & $e_{y}$ & $L$ & $l$ & $H$ & $b$ & $h$ & $c_{x}$ & $c_{y}$ & $d_{x}$ & $d_{y}$ \\
\hline 80 & 0 & 375 & 825 & 632 & 480 & 150 & 0 & 79 & 0 & 51 \\
\hline
\end{tabular}

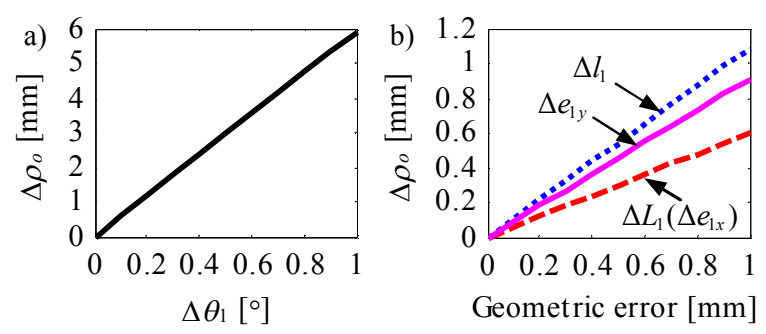

Fig. 5. Sensitivity analyses; a) variation of $\Delta \rho_{0}$ vs. $\Delta \varphi_{0}$ and b) variations of $\Delta \rho_{0}$ vs. geometric errors

\section{ZERO OFFSET IDENTIFICATION}

The zero-offset identification model is developed based on two adjacent measurement positions, $P_{k}$ and $P_{k+1}\left(1 \leq k \leq K^{-1}\right.$, and $K$ is the total number of measurement positions). As shown in Fig. 6, considering the position errors of $O_{1}$ and $O_{2}$, then $\overline{O_{1} O_{2}}$ and $\overline{O_{1}^{\prime} O_{2}^{\prime}}$ of $P_{k}$, denoted by $\lambda_{k}$ and $\boldsymbol{\rho}_{k}$, respectively, can be expressed as:

$$
\begin{gathered}
\lambda_{k}=\lambda_{k} \hat{\lambda}_{k}=\boldsymbol{r}_{k}-\boldsymbol{c}-\boldsymbol{d}, \\
\rho_{k}=\rho_{k} \hat{\boldsymbol{\rho}}_{k}=\boldsymbol{r}_{k}^{\prime}-\boldsymbol{c}^{\prime}-\boldsymbol{d}^{\prime},
\end{gathered}
$$

where $\lambda_{k}$ and $\hat{\lambda}_{k}$ are the length and unit orientation vector of $\lambda_{k} ; \rho_{k}$ and $\hat{\rho}_{k}$ are the length and unit orientation vector of $\boldsymbol{\rho}_{k} ; \boldsymbol{r}_{k}$ and $\boldsymbol{r}_{k}^{\prime}$ are the nominal and actual position vectors of $P_{k} ; \boldsymbol{c}^{\prime}$ is the position vector from $O$ to $O_{1}^{\prime} ; \boldsymbol{d}^{\prime}$ is the position vector from $O_{2}^{\prime}$ to $P_{k}^{\prime}$. Then, taking the first-order approximation of Eq. (14) yields:

$$
\Delta \lambda_{k} \hat{\lambda}_{k}+\lambda_{k} \Delta \hat{\lambda}_{k}=\Delta \boldsymbol{r}_{k}-\Delta \boldsymbol{r}_{M},
$$

where $\Delta \lambda_{k}$ and $\Delta \hat{\lambda}_{k}$ are the length error and orientation error vector of $\lambda_{k} ; \Delta \boldsymbol{r}_{k}$ is the position error vector of $P_{k} ; \Delta \boldsymbol{r}_{M}=\left(\Delta r_{M x} \Delta r_{M x}\right)^{\mathrm{T}}$ is the MAEMM; and we can obtain:

$$
\begin{gathered}
\Delta \boldsymbol{r}_{M}=\Delta \boldsymbol{c}+\Delta \boldsymbol{d}, \quad \Delta \boldsymbol{c}=\boldsymbol{c}^{\prime}-\boldsymbol{c}, \quad \Delta \boldsymbol{d}=\boldsymbol{d}^{\prime}-\boldsymbol{d}, \\
\Delta \lambda_{k}=\rho_{k}-\lambda_{k},
\end{gathered}
$$

where $\Delta \boldsymbol{c}^{\prime}$ and $\Delta \boldsymbol{d}^{\prime}$ are the position error vectors of $O_{1}$ and $O_{2}$, respectively.

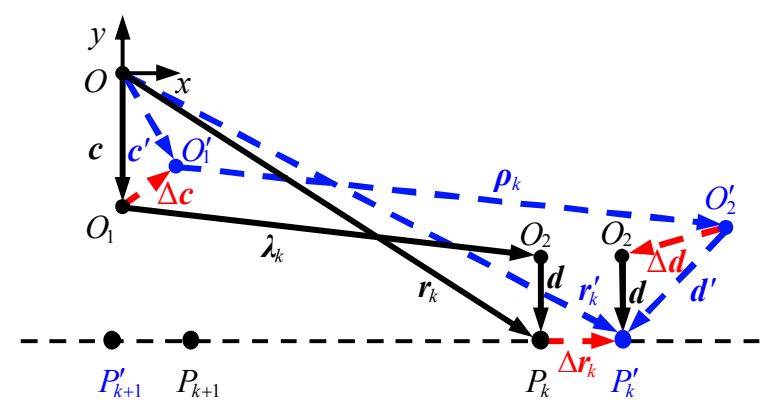

Fig. 6. Error model of the measuring mechanism Note: $O_{1}^{\prime}\left(O_{2}^{\prime}\right)$ is the actual rotation centre of the revolute joint $3(4) ; P_{k}\left(P_{k+1}\right)$ and $P_{k}^{\prime}\left(P_{k+1}^{\prime}\right)$ are the $k^{\text {th }}\left((k+1)^{\text {th }}\right)$ nominal and actual measurement positions, respectively

Taking dot product with $\hat{\lambda}_{k}^{\mathrm{T}}$ on the both sides of Eq. (16) (note that $\hat{\lambda}_{k} \perp \Delta \hat{\lambda}_{k}$ ) yields:

$$
\Delta \lambda_{k}=\hat{\lambda}_{k}^{\mathrm{T}}\left(\Delta \boldsymbol{r}_{k}-\Delta \boldsymbol{r}_{M}\right)
$$

Then substituting Eq. (12) and Eq. (18) into Eq. (19), we can obtain: 


$$
\rho_{k}-\lambda_{k}=\hat{\lambda}_{k}^{\mathrm{T}}\left(\boldsymbol{J}_{k}^{\prime} \Delta \boldsymbol{q}^{\prime}-\Delta \boldsymbol{r}_{M}\right)
$$

where $\boldsymbol{J}_{k}^{\prime}$ is the error transfer matrix $\boldsymbol{J}^{\prime}$ of $P_{k}$.

Rewriting Eq. (20) as:

$$
\rho_{k}-\lambda_{k}=\boldsymbol{g}_{k}^{\prime} \Delta \boldsymbol{p}
$$

where $\boldsymbol{g}_{k}^{\prime}=\hat{\lambda}_{k}^{\mathrm{T}}\left[\begin{array}{ll}\boldsymbol{J}_{k}^{\prime} & -\boldsymbol{I}\end{array}\right], \quad \boldsymbol{I}=\left[\begin{array}{ll}1 & 0 \\ 0 & 1\end{array}\right], \quad \Delta \boldsymbol{p}^{\prime}=\left(\begin{array}{c}\Delta \boldsymbol{q}^{\prime} \\ \Delta \boldsymbol{r}_{M}\end{array}\right)$.

We can also get Eq. (22) according to $P_{k+1}$ :

$$
\rho_{k+1}-\lambda_{k+1}=\boldsymbol{g}_{k+1}^{\prime} \Delta \boldsymbol{p}^{\prime},
$$

where $\boldsymbol{g}_{k+1}^{\prime}=\hat{\lambda}_{k+1}^{\mathrm{T}}\left[\begin{array}{ll}\boldsymbol{J}_{k+1} & -\boldsymbol{I}\end{array}\right] ; \rho_{k+1}$ and $\hat{\boldsymbol{\rho}}_{k+1}$ are the length and unit orientation vector of $\boldsymbol{\rho}_{k+1} ; \boldsymbol{\rho}_{k+1}$ is $\overline{O_{1}^{\prime} O_{2}^{\prime}}$ of $P_{k+1} ; \lambda_{k+1}$ and $\hat{\lambda}_{k+1}$ are the length and unit orientation vector of $\lambda_{k+1} ; \lambda_{k+1}$ is $\overline{O_{1} O_{2}}$ of $P_{k+1}$; and $\boldsymbol{J}_{k+1}^{\prime}$ is the error transfer matrix $\boldsymbol{J}^{\prime}$ of $P_{k+1}$.

Subtracting Eq. (22) with Eq. (21) leads to:

$$
\left(\rho_{k+1}-\rho_{k}\right)-\left(\lambda_{k+1}-\lambda_{k}\right)=\left(\boldsymbol{g}_{k+1}^{\prime}-\boldsymbol{g}_{k}^{\prime}\right) \Delta \boldsymbol{p}^{\prime} .
$$

Hence, the matrix form of the identification model can be expressed as:

$$
\Delta \lambda=\boldsymbol{G}^{\prime} \Delta \boldsymbol{p}^{\prime},
$$

where

$$
\Delta \lambda=\left(\begin{array}{c}
\left(\rho_{2}-\rho_{1}\right)-\left(\lambda_{2}-\lambda_{1}\right) \\
\vdots \\
\left(\rho_{k+1}-\rho_{k}\right)-\left(\lambda_{k+1}-\lambda_{k}\right) \\
\vdots \\
\left(\rho_{K}-\rho_{K-1}\right)-\left(\lambda_{K}-\lambda_{K-1}\right)
\end{array}\right), \quad \boldsymbol{G}^{\prime}=\left[\begin{array}{c}
\boldsymbol{g}_{2}^{\prime}-\boldsymbol{g}_{1}^{\prime} \\
\vdots \\
\boldsymbol{g}_{k+1}^{\prime}-\boldsymbol{g}_{k}^{\prime} \\
\vdots \\
\boldsymbol{g}_{K}^{\prime}-\boldsymbol{g}_{K-1}^{\prime}
\end{array}\right] .
$$

It is easy to prove that rank $\left(\boldsymbol{G}^{\prime}\right)=12$ if $K \geq 13$ provided that $\hat{\lambda}_{1}, \hat{\lambda}_{2}, \cdots, \hat{\lambda}_{K-1}$ and $\hat{\lambda}_{K}$ are not colinear, then $\Delta \boldsymbol{p}^{\prime}$ can be identified using the LS method:

$$
\Delta \boldsymbol{p}^{\prime}=\left[\left(\boldsymbol{G}^{\prime}\right)^{\mathrm{T}} \boldsymbol{G}^{\prime}\right]^{-1}\left(\boldsymbol{G}^{\prime}\right)^{\mathrm{T}} \Delta \boldsymbol{\lambda} .
$$

The singular value decomposition method is often used to study the identifiability, by which the identification matrix $\boldsymbol{G}^{\prime}$ can be rewritten as:

$$
\boldsymbol{G}^{\prime}=\boldsymbol{U}^{\prime} \boldsymbol{S}^{\prime}\left(\boldsymbol{V}^{\prime}\right)^{\mathrm{T}}
$$

where $\boldsymbol{U}^{\prime}$ and $\boldsymbol{V}^{\prime}$ are $(K-1) \times(K-1)$ and $12 \times 12$ matrixes, respectively, and each of them is composed of a set of standard orthogonal bases; $\boldsymbol{S}^{\prime}$ is a diagonal matrix composed of the singular values of $\boldsymbol{G}^{\prime}$. Hence, Eq. (25) can be rewritten as:

$$
\Delta \boldsymbol{p}^{\prime}=\sum_{t=1}^{12} \frac{\left(\boldsymbol{u}_{t}^{\prime}\right)^{\mathrm{T}} \Delta \lambda}{\sigma_{t}^{\prime}} \boldsymbol{v}_{t}^{\prime},
$$

where $\boldsymbol{u}_{t}^{\prime}$ is the standard orthogonal basis of $\boldsymbol{U}^{\prime} ; \boldsymbol{v}_{t}^{\prime}$ is the standard orthogonal basis of $\boldsymbol{V}^{\prime}$; $\sigma_{t}^{\prime}$ is the singular value of $\boldsymbol{G}^{\prime}$, and $\sigma_{1}^{\prime} \geq \sigma_{2}^{\prime} \geq \cdots \geq \sigma_{12}^{\prime}>0$.
The TSVD method can be used to improve the identifiability of $\Delta \boldsymbol{p}^{\prime}$ by simply truncating the summation in Eq. (27) at an upper limit $t \leq 12$ before the small singular values start to dominate. However, if the zero offsets are identified together with too many geometric errors, the upper limit of $t$ will be too large and then the TSVD may be performed like the LS that cannot overcome the nonlinear illconditioning problem of Eq. (25). Since it has been proved in Section 3 that the position accuracy is more sensitive to the zero offset than the geometric errors, the nonlinear ill-conditioning problem can be directly solved to some extent by neglecting the identification of the geometric errors according to the TSVD method, i.e. by substituting Eq. (13) and Eq. (18) into Eq. (19), then the identification model can be degenerated into the following form:

$$
\Delta \boldsymbol{\lambda}=\boldsymbol{G} \Delta \boldsymbol{p}, \boldsymbol{G}=\left[\begin{array}{c}
\boldsymbol{g}_{2}-\boldsymbol{g}_{1} \\
\vdots \\
\boldsymbol{g}_{k+1}-\boldsymbol{g}_{k} \\
\vdots \\
\boldsymbol{g}_{K}-\boldsymbol{g}_{K-1}
\end{array}\right],\left\{\begin{array}{l}
\Delta \boldsymbol{p}=\left(\begin{array}{ll}
\Delta \boldsymbol{q} & \Delta \boldsymbol{r}_{M}
\end{array}\right)^{\mathrm{T}} \\
\boldsymbol{g}_{k}=\hat{\lambda}_{k}^{\mathrm{T}}\left[\begin{array}{ll}
\boldsymbol{J}_{k} & -\boldsymbol{I}
\end{array}\right] \\
\boldsymbol{g}_{k+1}=\hat{\lambda}_{k+1}^{\mathrm{T}}\left[\begin{array}{ll}
\boldsymbol{J}_{k+1} & -\boldsymbol{I}
\end{array}\right]
\end{array}\right.
$$

where $\boldsymbol{J}_{k}$ and $\boldsymbol{J}_{k+1}$ are the error transfer matrix $\boldsymbol{J}$ of $P_{k}$ and $P_{k+1}$, respectively.

It can also be proved that $\operatorname{rank}(\boldsymbol{G})=4$ if $K \geq 5$ provided that $\hat{\lambda}_{1}, \hat{\lambda}_{2}, \cdots, \hat{\lambda}_{K-1}$ and $\hat{\lambda}_{K}$ are not colinear. Hence, $\Delta p$ can be calculated by

$$
\Delta \boldsymbol{p}=\sum_{t=1}^{4} \frac{\left(\boldsymbol{u}_{t}\right)^{\mathrm{T}} \Delta \boldsymbol{\lambda}}{\sigma_{t}} \boldsymbol{v}_{t},
$$

where $\boldsymbol{u}_{t}, \boldsymbol{v}_{t}$ and $\sigma_{t}$ are the standard orthogonal bases and singular value derived from the SVD format of the identification matrix $\boldsymbol{G}$.

Since we neglect the identification of the geometric errors, the accuracy of $\Delta \theta_{i}$ solved by Eq. (29) may be slightly decreased even though the nonlinear ill-conditioning problem can be solved. To improve the accuracy, the following aspects are considered: (1) the source errors should be identified multiple times; (2) the measuring mechanism is used as a metrology device and its measurement accuracy can be improved with the decrease of $\Delta \boldsymbol{r}_{M}$; (3) $\Delta \boldsymbol{r}_{M}$ is independent of the source errors of the robot, and the smaller $\Delta \boldsymbol{r}_{M}$ the better the identifiability of $\Delta \theta_{i}$. Based on these considerations, the identification model can be modified as follows.

For the $1^{\text {st }}$ identification, we use Eq. (29) to identify $\Delta p$; for the $j^{\text {th }}(j \geq 2)$ identification, by modifying Eq. (20) with the former identification result of $\Delta \boldsymbol{r}_{M}$ as shown in Eq. (30), then the $j^{\text {th }}$ identification model can be redeveloped: 


$$
\rho_{k}-\left\|\boldsymbol{f}_{k}^{(j)}\right\|=\frac{\left(\boldsymbol{f}_{k}^{(j)}\right)^{\mathrm{T}}}{\left\|\boldsymbol{f}_{k}^{(j)}\right\|}\left(\boldsymbol{J}_{k} \Delta \boldsymbol{q}^{(j)}-\Delta \boldsymbol{r}_{M}^{(j)}\right),
$$

where $\boldsymbol{f}_{k}^{(j)}=\boldsymbol{f}_{k}^{(j-1)}+\Delta \boldsymbol{r}_{M}^{(j-1)}$, and $\boldsymbol{f}_{k}^{(1)}=\boldsymbol{\lambda}_{k}$.

\section{OPTIMAL MEASUREMENT POSITIONS AND ERROR COMPENSATION STRATEGY}

The identification of $\Delta p$ requires the moving platform to undergo $K \geq 5$ measurement positions; meanwhile, these positions should converge to the boundaries of the workspace where the highest signal-to-noise ratio be may achieved. In addition, the moving platform should experience all the controllable degrees of freedom. As shown in Fig. 7, the most straightforward way is to choose $n$ evenly spaced positions on each of the upper and lower boundaries of the workspace.

Since the identifiability can be improved with the decrease of the condition number of the identification matrix, to make the measurements in a time-effective manner, the selection problem of the optimal measurement positions can be solved by minimizing $n, \kappa_{1}$ and $\kappa_{m}$ subject to a given threshold $\varepsilon_{0}$ defined as the relative difference between $\kappa_{1}$ and $\kappa_{m} v s$. $n$, i.e.

$$
\min \left\{n, \kappa_{1}, \kappa_{m}\right\} \quad \text { s.t. } \varepsilon=\frac{\left|\kappa_{1}-\kappa_{m}\right|}{\kappa_{1}} \leq \varepsilon_{0}, n \geq 3,
$$

where $\kappa_{1}$ denotes the condition number of the first identification; and $\kappa_{m}$ denotes the mean condition number of the remaining identifications.

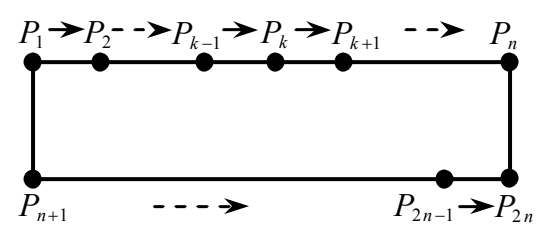

Fig. 7. Optimal measurement positions

After $\Delta \theta_{i}$ of the $j^{\text {th }}$ and $(j+1)^{\text {th }}$ identifications are obtained, the position error of the robot can be reduced in an iterative manner by compensating the kinematic model in the robot controller with the identification results of $\Delta \theta_{i}$ until the compensation accuracy $\mu$ defined as follows converges within a given threshold $\mu_{0}$ :

$$
\mu=\sqrt{\left[\left(\Delta \theta_{1}^{(j+1)}-\Delta \theta_{1}^{(j)}\right)^{2}+\left(\Delta \theta_{2}^{(j+1)}-\Delta \theta_{2}^{(j)}\right)^{2}\right] / 2} .
$$

Then the compensation value of $\Delta \theta_{i}$ is:

$$
\Delta \theta_{i}=\Delta \theta_{i}^{(1)}+\Delta \theta_{i}^{(2)}+\cdots+\Delta \theta_{i}^{(j)}+\cdots+\Delta \theta_{i}^{(m)},
$$

where $m$ is the compensation number.

\section{SIMULATION ANALYSES}

In this section, simulations are carried out to investigate the accuracy and robustness of the zerooffset calibration method in depth.

\subsection{Simulation Parameters}

The given source errors are listed in Table 2. This is because: 1) the investigation of the identification accuracy requires the given values of $\Delta \theta_{i}$ to cover a certain range; 2) the different attainable geometric accuracies of the robot should be considered; 3) the MAEMM can be roughly measured, and $\Delta r_{M x}$ and $\Delta r_{M y}$ are about $1 \mathrm{~mm}$ and $0.5 \mathrm{~mm}$, respectively.

Given $\Delta \boldsymbol{r}_{M}^{(j)}=\left(\begin{array}{ll}1 & 0.5\end{array}\right)^{\mathrm{T}}(2 \leq j \leq m)$, it can be seen from Fig. 8 that, for each simulation group, $\kappa_{1}$ and $\kappa_{m}$ both monotonically increase with the increase of $n$. Meanwhile, $\kappa_{m}$ is less than $\kappa_{1}$ corresponding to the same $n$, meaning that the identifiability can be slightly improved by using Eq. (30). Furthermore, given $\varepsilon_{0}=0.01$, it can also be seen that the minimum $n$ is 3, which leads to $K=2 n=6$ optimal measurement positions.

Based on the optimal positions, $\left(\lambda_{k}-\lambda_{k+1}\right)$ can be obtained by the inverse positional analysis, and $\left(\rho_{k}-\rho_{k+1}\right)$ can be derived from the forward positional model containing the source errors and considering the measurement errors. Then $\Delta \theta_{i}$ can be calibrated using the proposed method.

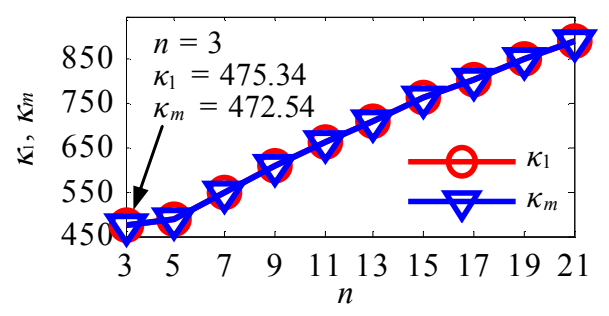

Fig. 8. Variations of $\kappa_{1}$ and $\kappa_{m}$ vs. $n$

In the calibration, the measurement errors are mainly caused by the linear scale and servo motor, which can be reasonably set as follows.

Since the maximum measurement error of the linear scale is $\pm\left(3+l_{0} / 1000\right) \times 10^{-3} \mathrm{~mm}\left(l_{0}\right.$ is the measuring range of the linear scale and $l_{0}=350 \mathrm{~mm}$ ); meanwhile, the output of the reading head can be reset after each measurement, the measurement error of the linear scale corresponding to $P_{k}$, denoted by $\omega_{k}$, can be set as the Gaussian distributed error with mean 0 and variance $\omega^{2}$, and $\omega$ can be calculated by:

$$
\omega=\frac{1}{3}\left(3+\frac{l_{0}}{1000}\right) \times 10^{-3} .
$$


Table 2. Set values of the source errors

\begin{tabular}{|c|c|c|c|c|c|c|c|c|c|c|c|c|}
\hline \multirow{2}{*}{ Group } & \multicolumn{2}{|c|}{ Zero offset $\left[{ }^{\circ}\right]$} & \multicolumn{8}{|c|}{ Geometric error [mm] } & \multicolumn{2}{|c|}{ MAEMM [mm] } \\
\hline & $\Delta \theta_{1}$ & $\Delta \theta_{2}$ & $\Delta e_{1 x}$ & $\Delta e_{1 y}$ & $\Delta e_{2 x}$ & $\Delta e_{2 y}$ & $\Delta L_{1}$ & $\Delta L_{2}$ & $\Delta l_{1}$ & $\Delta l_{2}$ & $\Delta r_{M x}$ & $\Delta r_{M v}$ \\
\hline 1 & 2 & -1 & \multirow{3}{*}{0.03} & \multirow{3}{*}{-0.02} & \multirow{3}{*}{-0.02} & \multirow{3}{*}{0.01} & \multirow{3}{*}{0.01} & \multirow{3}{*}{0.02} & \multirow{3}{*}{-0.03} & \multirow{3}{*}{-0.02} & \multirow{6}{*}{1} & \multirow{6}{*}{0.5} \\
\hline 2 & -1 & 0.5 & & & & & & & & & & \\
\hline 3 & 0.5 & 0.25 & & & & & & & & & & \\
\hline 4 & 2 & -1 & \multirow{3}{*}{0.003} & \multirow{3}{*}{-0.002} & \multirow{3}{*}{-0.002} & \multirow{3}{*}{0.001} & \multirow{3}{*}{0.001} & \multirow{3}{*}{0.002} & \multirow{3}{*}{-0.003} & \multirow{3}{*}{-0.002} & & \\
\hline 5 & -1 & 0.5 & & & & & & & & & & \\
\hline 6 & 0.5 & 0.25 & & & & & & & & & & \\
\hline
\end{tabular}

Table 3. Simulation results of $\Delta \theta_{i}$ and $\Delta \rho_{0}$

\begin{tabular}{|c|c|c|c|c|c|}
\hline Group & $\begin{array}{l}\text { Zero } \\
\text { offset }\end{array}$ & $\begin{array}{c}\text { Compensation } \\
\text { value }\left[^{\circ}\right]\end{array}$ & $\delta\left[^{\circ}\right]$ & $\begin{array}{c}\Delta \rho_{0} \\
\text { (before) } \\
{[\mathrm{mm}]}\end{array}$ & $\begin{array}{c}\Delta \rho_{0} \\
\text { (after) } \\
{[\mathrm{mm}]}\end{array}$ \\
\hline \multirow{2}{*}{1} & $\Delta \theta_{1}$ & 2.033 & 0.033 & \multirow{2}{*}{10.933} & \multirow{2}{*}{0.402} \\
\hline & $\Delta \theta_{2}$ & -0.957 & 0.043 & & \\
\hline \multirow{2}{*}{2} & $\Delta \theta_{1}$ & -1.049 & 0.049 & \multirow{2}{*}{5.482} & \multirow{2}{*}{0.423} \\
\hline & $\Delta \theta_{2}$ & 0.463 & 0.037 & & \\
\hline \multirow{2}{*}{3} & $\Delta \theta_{1}$ & 0.536 & 0.036 & \multirow{2}{*}{3.796} & \multirow{2}{*}{0.434} \\
\hline & $\Delta \theta_{2}$ & 0.297 & 0.047 & & \\
\hline \multirow{2}{*}{4} & $\Delta \theta_{1}$ & 2.024 & 0.024 & \multirow{2}{*}{10.896} & \multirow{2}{*}{0.228} \\
\hline & $\Delta \theta_{2}$ & -0.978 & 0.022 & & \\
\hline \multirow{2}{*}{5} & $\Delta \theta_{1}$ & -1.025 & 0.025 & \multirow{2}{*}{5.5195} & \multirow{2}{*}{0.256} \\
\hline & $\Delta \theta_{2}$ & 0.474 & 0.026 & & \\
\hline \multirow{2}{*}{6} & $\Delta \theta_{1}$ & 0.476 & 0.024 & \multirow{2}{*}{3.798} & \multirow{2}{*}{0.239} \\
\hline & $\Delta \theta_{2}$ & 0.225 & 0.025 & & \\
\hline
\end{tabular}

Considering that the number of pulses per revolution of the servo motor is $1 \times 10^{4}$ and the maximum number of pulse error sper revolution is 4 , the motion error of the servo motor corresponding to $P_{k}$, denoted by $\xi_{k}$, can also be set as the Gaussian distributed error with mean 0 and variance $\xi^{2}$, and $\xi$ can be calculated by:

$$
\xi=\frac{1}{3}\left(\frac{4}{\eta \times 10^{4}}\right) \times 360^{\circ},
$$

where $\eta=20$ is the reduction ratio of the reducer.

\subsection{Simulation Results and Discussion}

Given $\mu_{0}=0.1^{\circ}$, the compensation value of $\Delta \theta_{i}$, the absolute difference between the set and compensation values of $\Delta \theta_{i}$, denoted by $\delta$, and $\Delta \rho_{0}$ before and after calibration are listed in Table 3 . It can be seen that $\delta$ is around $0.040^{\circ}$ in the first three groups and $0.025^{\circ}$ in the last three groups. This indicates that the identification accuracy is invariant with the set values of $\Delta \theta_{i}$, and that it can be slightly improved with the decrease of the geometric errors. Furthermore, $\Delta \rho_{0}$ can be significantly reduced after calibration, and the maximum $\Delta \rho_{0}$ after calibration is $0.434 \mathrm{~mm}$ in the first three groups and $0.256 \mathrm{~mm}$ in the last three groups.

As shown in Table 4, for each group, the maximum absolute distance error, denoted by $\Delta \rho_{\max }$, of the six optimal measurement positions can be reduced to a certain value after calibration. Since these positions are along the boundaries of the workspace where the position errors usually tend to be much larger than those of the internal positions, we can infer that the position accuracy throughout the workspace of the robot can be well improved after the calibration.

Table 4. $\Delta \rho_{\max }$ before and after calibration

\begin{tabular}{lcccccc}
\hline Group & 1 & 2 & 3 & 4 & 5 & 6 \\
\hline $\begin{array}{l}\Delta \rho_{\max } \\
\text { (before) }\end{array}$ & 12.906 & 6.418 & 4.672 & 12.868 & 6.455 & 4.663 \\
[mm] & & & & & & \\
\hline $\begin{array}{l}\Delta \rho_{\max } \\
\text { (after) } \\
\text { [mm] }\end{array}$ & 0.493 & 0.531 & 0.533 & 0.279 & 0.314 & 0.293 \\
\hline
\end{tabular}

Table 5 shows the absolute differences between the set values and identification results of $\Delta r_{M x}$ and $\Delta r_{M y}$, denoted by $\delta_{M x}$ and $\delta_{M y}$, respectively. It can be seen that, similar to the identification results of $\Delta \theta_{i}$, the identification accuracies of $\Delta r_{M x}$ and $\Delta r_{M y}$ are scarcely affected by the set values of $\Delta \theta_{i}$, while they can be slightly improved with the decrease of the geometric errors of the robot.

Table 5. Absolute differences between the set values and identification results of $\Delta r_{M x}$ and $\Delta r_{M y}$

\begin{tabular}{lcccccc}
\hline Group & 1 & 2 & 3 & 4 & 5 & 6 \\
\hline$\delta_{M x}[\mathrm{~mm}]$ & 0.069 & 0.074 & 0.071 & 0.044 & 0.051 & 0.047 \\
\hline$\delta_{M y}[\mathrm{~mm}]$ & 0.040 & 0.045 & 0.042 & 0.022 & 0.028 & 0.024 \\
\hline
\end{tabular}

As shown in Table 6, of each group is about 472.50 , which is almost the same as $\kappa_{m}=472.54$ and less than $\kappa_{1}=475.34$ as shown in Fig. 8, further verifying that the identifiability of the identification 

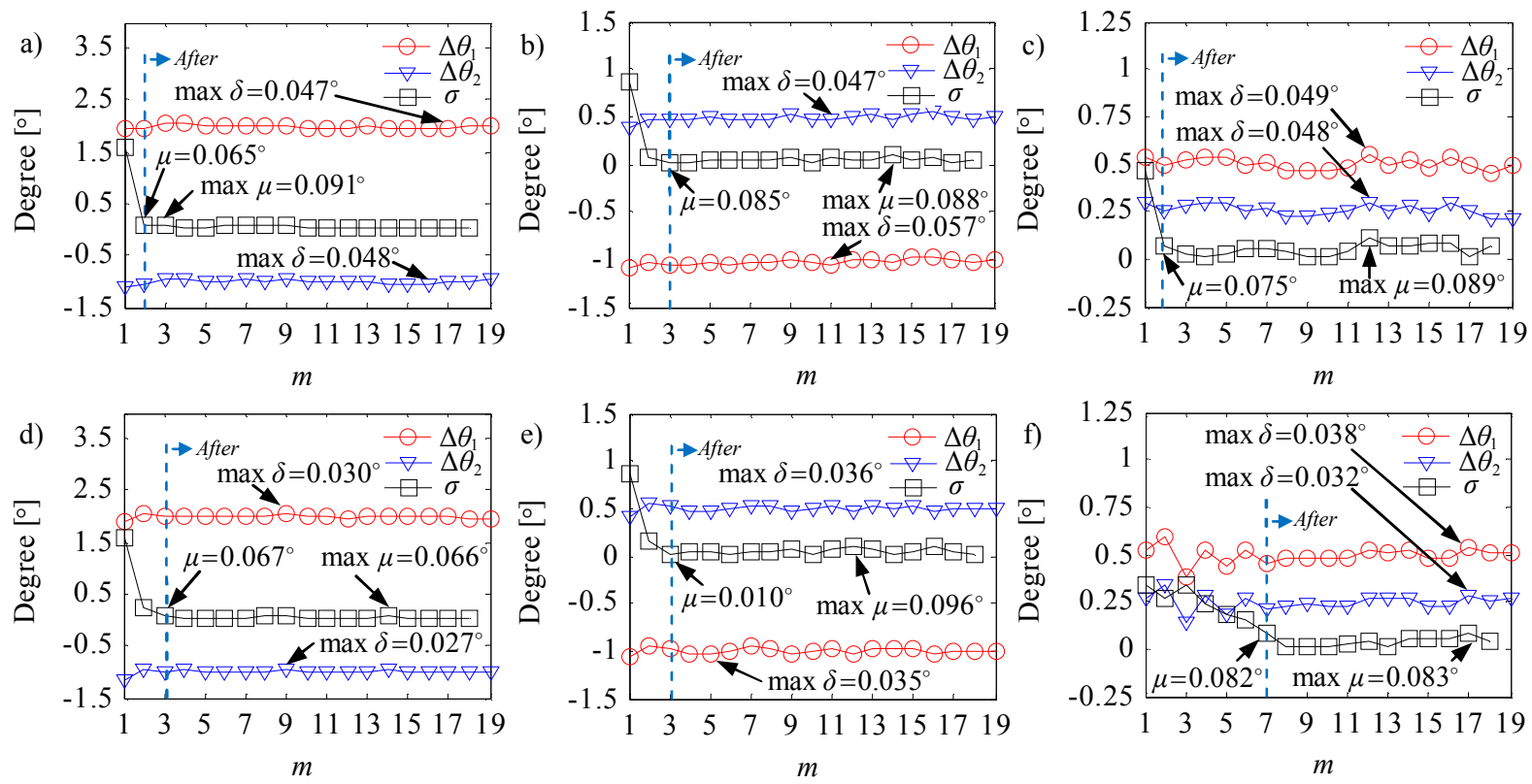

Fig. 9. Robustness analyses; a) group 1; b) group 2; c) group 3; d) group 4; e) group 5; and f) group 6

model modified using Eq. (30) can be slightly improved.

Table 6. Mean condition number

\begin{tabular}{ccccccc}
\hline Group & 1 & 2 & 3 & 4 & 5 & 6 \\
\hline$\kappa_{m}$ & 472.47 & 472.49 & 472.49 & 472.50 & 472.51 & 472.50 \\
\hline
\end{tabular}

Table 7. Experimental results of $\Delta \theta_{i}$ and $\Delta \rho_{0}$

\begin{tabular}{|c|c|c|c|c|c|}
\hline Group & $\begin{array}{l}\text { Zero- } \\
\text { offset }\end{array}$ & $\begin{array}{c}\text { Compensation } \\
\text { value }\left[{ }^{\circ}\right]\end{array}$ & $\delta\left[^{\circ}\right]$ & $\begin{array}{c}\Delta \rho_{0} \\
\text { (before) } \\
{[\mathrm{mm}]}\end{array}$ & $\begin{array}{c}\Delta \rho_{0} \\
\text { (after) } \\
\text { [mm] }\end{array}$ \\
\hline \multirow{2}{*}{1} & $\Delta \theta_{1}$ & 1.929 & 0.071 & \multirow{2}{*}{12.062} & \multirow{2}{*}{0.732} \\
\hline & $\Delta \theta_{2}$ & -0.924 & 0.076 & & \\
\hline \multirow{2}{*}{2} & $\Delta \theta_{1}$ & -0.935 & 0.065 & \multirow{2}{*}{6.964} & \multirow{2}{*}{0.605} \\
\hline & $\Delta \theta_{2}$ & 0.572 & 0.072 & & \\
\hline \multirow{2}{*}{3} & $\Delta \theta_{1}$ & 0.569 & 0.069 & \multirow{2}{*}{4.945} & \multirow{2}{*}{0.711} \\
\hline & $\Delta \theta_{2}$ & 0.175 & 0.075 & & \\
\hline
\end{tabular}

Further research is performed to evaluate the robustness of the identification model. The variations of the compensation value of $\Delta \theta_{i}$ and the defined compensation accuracy $\mu$ versus $m$ of each group are presented in Fig. 9. For each group, it can be observed that the compensation values of $\Delta \theta_{1}$ and $\Delta \theta_{2}$ both fluctuate slightly, but they can converge to different values with the increase of $m$. Furthermore, $\delta$ is less than $0.060^{\circ}$ in the first three groups and $0.040^{\circ}$ in the last three groups, and $\mu$ of each group after its value reduces to less than $\mu_{0}=0.1^{\circ}$ for the first time is between $0^{\circ}$ to $0.1^{\circ}$. These observations indicate that the identification model has good robustness.

\section{EXPERIMENTAL VERIFICATION}

Experiments are carried out on the 2-DOF parallel robot with the repeatability of $\pm 0.05 \mathrm{~mm}$ over its workspace to verify the effectiveness of the zerooffset calibration method.

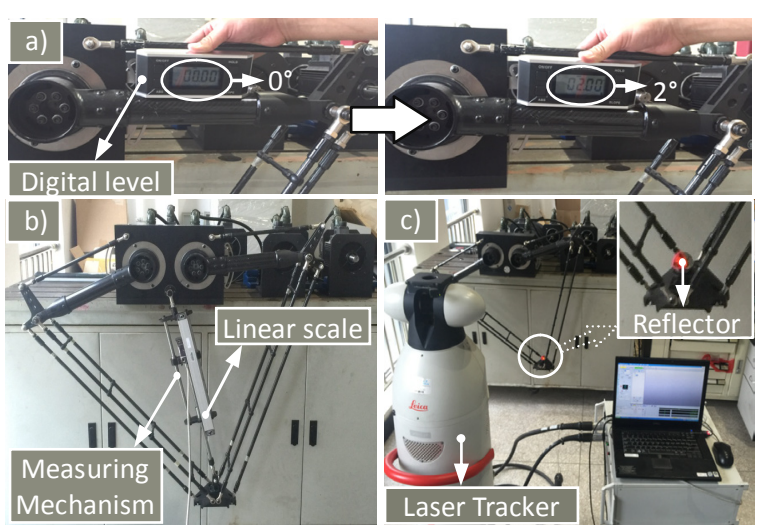

Fig. 10. Experiment set-ups; a) zero offset adjustment set-up; b) calibration set-up; and c) verification set-up

As shown in Fig. 10a, in order to compare the experiments with the simulations, a digital level with the maximum observed deviation of $0.1^{\circ}$ is used to adjust the two active proximal links to the horizontal position before each experiment, and then 
$\Delta \theta_{1}$ and $\Delta \theta_{2}$ can be roughly regarded as $0^{\circ}$. After that, $\Delta \theta_{1}$ and $\Delta \theta_{2}$ are set as listed in the first three groups of Table 2, respectively, by driving the two active proximal links to the corresponding positions. Having built the calibration set-up as shown in Fig. $10 \mathrm{~b}$, the experiments of the zero-offset calibration can be implemented, and the position errors before and after calibration are measured with a LEICA AT901 laser tracker with the maximum observed deviation of $0.016 \mathrm{~mm}$ as shown in Fig. 10c.

Table 8. Identification results of $\Delta \mathrm{rMx}$ and $\Delta \mathrm{rMy}$

\begin{tabular}{lccc}
\hline Group & 1 & 2 & 3 \\
\hline$\Delta r_{M x}[\mathrm{~mm}]$ & 0.842 & 0.835 & 0.852 \\
\hline$\Delta r_{M y}[\mathrm{~mm}]$ & 0.592 & 0.576 & 0.583 \\
\hline
\end{tabular}

Likewise, given $\mu_{0}=0.1^{\circ}$, the experimental results are listed in Tables 7 and 8, from which we can determine that, similar to the simulation results, the identification accuracy is invariant with the set values of $\Delta \theta_{i}$, and the $\Delta \rho_{0}$ of each group can be significantly reduced after calibration. We can also determine that the maximum $\delta$ and $\Delta \rho_{0}$ after calibration are $0.076^{\circ}$ and $0.732 \mathrm{~mm}$, respectively, which are slightly larger than $0.049^{\circ}$ and $0.434 \mathrm{~mm}$ of the simulations. Since it has been proved via the simulation analyses that the identifiability will decrease with the increase of the geometric errors, the slight decrease of the identification accuracy in the experiments is due to the fact that the actual geometric errors of the robot are larger than those given in the simulations.

As shown in Fig. 11, in order to carry out deeper investigation on the position accuracy, the absolute distance error, denoted by $\Delta \rho$, before and after calibration of $K=42$ evenly spaced measurement positions along the boundaries of the workspace are measured by the laser tracker, and the results are presented in Fig. 12. Furthermore, the maximum position error $\Delta \rho_{\max }$ of these positions are listed in Table 9. It can be seen that the maximum position error along the workspace of each group can also be significantly reduced to less than $0.85 \mathrm{~mm}$ after the calibration.

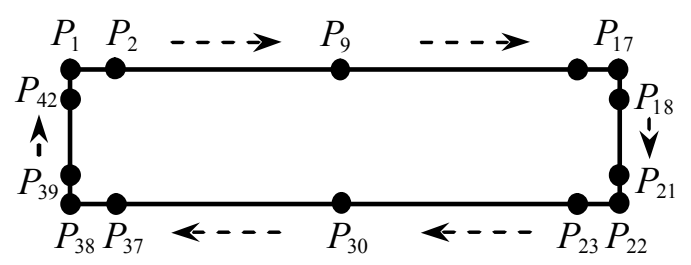

Fig. 11. The 42 measurement positions
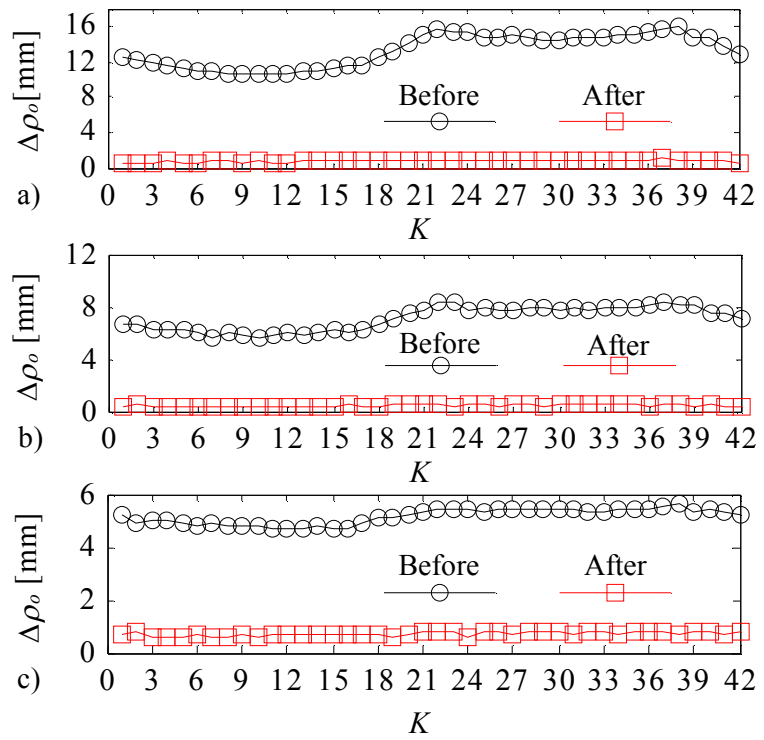

Fig. 12. $\Delta \rho$ before and after calibration; a) group 1; b) group 2; and c) group 3

Table 9. $\Delta \rho_{\max }$ before and after calibration

\begin{tabular}{lccc}
\hline Group & 1 & 2 & 3 \\
\hline$\Delta \rho_{\max }$ (before ) $[\mathrm{mm}]$ & 16.901 & 8.326 & 5.448 \\
\hline$\Delta \rho_{\max }$ (after) $[\mathrm{mm}]$ & 0.847 & 0.623 & 0.825 \\
\hline
\end{tabular}

\section{CONCLUSIONS}

To realize the rapid and automatic zero offset calibration of a 2-DOF parallel robot, a measuring mechanism is designed, and based on which a zerooffset calibration method is proposed in this paper. Compared with large measurement devices, the measuring mechanism is more convenient to use and it can make the measurements in a time and cost-effective manner. By using the TSVD method, the nonlinear ill-conditioning problem of the identification model can be solved. The identification model modified with the former identification result of the MAEMM can help to improve the identifiability of the zero offsets. The optimization approach for selecting measurement positions is able to maximize the measurement efficiency and further improve the identifiability. The simulation and experimental results of the calibration show that the identification model has good identifiability and robustness, and the position error after calibration can be significantly reduced. The proposed measuring mechanism and zero offset calibration method are also useful for the kinematic calibration of other planar or spatial parallel robots. It should be noted that since the spatial parallel 
robots usually have cylindrical workspaces, if the measuring mechanism is used for the calibration of these parallel robots, its two revolute joints should be replaced by universal or spherical joints, so that the measurement positions can be more reasonably selected in those cylindrical workspaces.

\section{ACKNOWLEDGEMENTS}

This work is supported by the National Natural Science Foundation of China (Grant No. 51475320 and 51420105007), and the Key Technologies R \& D Program of Tianjin (Grant No. 15ZXZNGX00220).

\section{REFERENCES}

[1] Clavel, R. (1988). Delta, a fast robot with parallel geometry. Proceedings of the $18^{\text {th }}$ International Symposium on Industrial Robots, p. 91-100.

[2] Pierrot, F., Nabat, V., Company, O., Krut, S., Poignet, P. (2009). Optimal design of a 4-DOF parallel manipulator: From academia to industry. IEEE Transactions on Robotics, vol. 25, no. 2, p. 213-224, DOI:10.1109/TR0.2008.2011412.

[3] Jin, Y., Chen, I.M., Yang, G.L. (2009). Kinematic design of a family of 6-DOF partially decoupled parallel manipulators. Mechanism and Machine Theory, vol. 44, no.5, p. 912-922, D0l:10.1016/j.mechmachtheory.2008.06.004.

[4] Cheng, G., Xu, P., Yang, D.H., Li, H., Liu, H.G. (2013). Analysing kinematics of a novel 3CPS parallel manipulator based on rodrigues parameters. Strojniski vestnik - Journal of Mechanical Engineering, vol. 59, no. 5, p. 291-300, DOI:10.5545/sv-jme.2012.727.

[5] Hao, G.B., Yu, J.J. (2016). Design, modelling and analysis of a completely-decoupled XY compliant parallel manipulator. Mechanisms and Machine Theory, vol. 102, p. 179-195, D0I:10.1016/j.mechmachtheory.2016.04.006.

[6] Hao, G.B., Kong, X.W. (2012). A novel large-range XY compliant parallel manipulator with enhanced out-of-plane stiffness. Journal of Mechanical Design, vol. 134, no. 6, p. 061009-1061009-9, D0l:10.1115/1.4006653.

[7] Huang, T., Liu, S.T., Mei, J.P., Chetwynd, D.G. (2013). Optimal design of a 2-DOF pick-and-place parallel robot using dynamic performance indices and angular constraints. Mechanisms and Machine Theory, vol. 70, p. 246-253, D0l:10.1016/j. mechmachtheory.2013.07.014.

[8] Cammarata, A. (2015). Optimized design of a large-workspace 2-DOF parallel robot for solar tracking systems. Mechanisms and Machine Theory, vol. 83, p. 175-186, D0l:10.1016/j. mechmachtheory.2014.09.012.

[9] Choi, Y., Cheong, J., Jin, H.K., Do, H.M. (2016). Zero-offset calibration using a screw theory. International Conference on Ubiquitous Robots and Ambient Intelligence, p. 526-528, DOI:10.1109/URAI.2016.7625770.

[10] Sun, Y.H., Wang, L., Mei, J.P., Zhang, W.C., Liu, Y. (2013). Zero calibration of delta robot based on monocular vision. Journal of Tianjin University, vol. 46, no. 3, p. 239-243. (in Chinese)
[11] Jáuregui, J.C., Hernández, E.E., Ceccarelli, M., López-Caju n, C., García, A. (2013). Kinematic calibration of precise 6-DOF Stewart platform-type positioning systems for radio telescope applications. Frontiers of Mechanical Engineering, vol. 8, no. 3, p. 252-260, DOI:10.1007/s11465-013-0249-7.

[12] Jin, Y., Chen I.M. (2006). Effects of constraint errors on parallel manipulators with decoupled motion. Mechanism and Machine Theory, vol. 41, no. 8, p. 912-928, D0l:10.1016/j. mechmachtheory.2006.03.012.

[13] Pan, B.Z., Song Y.M., Wang P.F., Dong, G., Sun, T. (2014). Laser tracker based rapid home position calibration of a hybrid robot. Journal of Mechanical Engineering, vol. 50, no. 1, p. 3137, D0I:10.3901/JME.2014.01.031. (in Chinese)

[14] Cheng, G., Gu, W., Li, J., Tang, Y.P. (2011). Overall structure calibration of 3-UCR parallel manipulator based on quaternion method. Strojniski vestnik - Journal of Mechanical Engineering, vol. 57, no. 10, p. 719-729, D0l:10.5545/sv-jme.2010.167.

[15] Last, P., Budde, C., Hesselbach, J. (2005). Self-calibration of the HEXA-parallel-structure. IEEE International Conference on Automation Science and Engineering, p. 393-398, DOI:10.1109/COASE.2005.1506801.

[16] Majarena, A.C., Santolaria, J., Samper, D., Aguilar, J.J. (2010). An overview of kinematic and calibration models using internal/external sensors or constraints to improve the behavior of spatial parallel mechanisms. Sensors, vol. 10, no. 11, p. 10256-10297, D0l:10.3390/s101110256.

[17] Rauf, A., Pervez, A., Ryu, J. (2006). Experimental results on kinematic calibration of parallel manipulators using a partial pose measurement device. IEEE Transactions on Robotics, vol. 22, no. 2, p. 379-344, D0I:10.1109/TR0.2006.862493.

[18] Kovac, I., Klein, A. (2002). Apparatus and a procedure to calibrate coordinate measuring arms. Strojniški vestnik Journal of Mechanical Engineering, vol. 48, no. 1, p. 17-32.

[19] Cedilnik, M., Sokovic, M., Jurkovic, J. (2006). Calibration and checking the geometrical accuracy of a CNC machine-tool. Strojniški vestnik - Journal of Mechanical Engineering, vol. 52, no. 11, p. 752-762.

[20] Takeda, Y., Gang S., Funabashi, H. (2004). A DBB-based kinematic calibration method for in-parallel actuated mechanism using a Fourier series. Journal of Mechanical Design, vol. 126, no. 5, p. 856-865, Dol:10.1115/1.1767822.

[21] Tian, W.J., Yin, F.W., Liu, H.T., Li, J.H., Li, Q., Huang, T., Chetwynd, D.G. (2016). Kinematic calibration of a 3-DOF spindle head using a double ball bar. Mechanism and Machine Theory, vol. 102, p. 167-178, D0l:10.1016/j. mechmachtheory.2016.04.008.

[22] Hoerl, A.E., Kennard, R.W. (2000). Ridge regression: biased estimation for nonorthogonal problems. Technometrics, vol. 42, no. 1, p. 80-86, D0l:10.1080/00401706.2000.10485983.

[23] Hansen, P.C., O'Leary, D.P. (1993). The use of the L-curve in the regularization of discrete ill-posed problems. SIAM Journal on Scientific Computing, vol. 14, no. 6, p. 1487-1503, DOI:10.1137/0914086.

[24] Yang, Z.Y., Wu, J., Mei, J.P. (2007). Motor-mechanism dynamic model based neural network optimized computed torque control of a high speed parallel manipulator. Mechatronics, vol. 17, no. 7, p. 381-390, Dol:10.1016/j. mechatronics.2007.04.009. 